\title{
Techniques used to calculate shale fractal dimensions involve uncertainties and imprecisions that require more careful consideration
}

\author{
David A. $\operatorname{Wood}^{\circledR *}$ \\ DWA Energy Limited, Lincoln, United Kingdom
}

Keywords:

Shale fractal dimensions

adsorption isotherm analysis

curve fitting uncertainties

optimization setups

pore-scale distributions

organic-rich shales

Cited as:

Wood, D. A. Techniques used to calculate shale fractal dimensions involve uncertainties and imprecisions that require more careful consideration. Advances in Geo-Energy Research, 2021, 5(2): 153-165, doi: 10.46690/ager.2021.02.05

\begin{abstract}
:
Surface roughness of shales has a key influence on the petroleum resources they are able to store and the fraction of them that can be recovered. The fractal dimension quantifies the degree of roughness and is influenced primarily by the pore surfaces within the shale that typically include micro-, meso- and macro-pores. Isotherms generated by gas adsorption experiments are the common data source used to derive estimates of fractal dimension. The Frenkel-Halsey-Hill fractal technique is the most widely applied fractal dimension estimation method. Other methods can derive fractal dimension from isotherm data but typically the values they generate are different from the Frenkel-Halsey-Hill derived fractal dimension values. Moreover, those differences can vary significantly depending on the type of shales involved. Those shales displaying more complex pore-scale distributions including extensive micro-porosity components tend to be associated with the greatest discrepancies. A comparison of three fractal dimension calculation methods applied to shales reveals aspects of their calculation and interpretation methods that explain the differences in the fractal dimension values they generate. This study identifies the uncertainties that should be taken into account when applying the methods and the appropriate curve fitting optimization configurations that should be evaluated. Taking these factors into account leads to more realistic selections of appropriate fractal dimension values from gas adsorption isotherms of organic-rich shales.
\end{abstract}

\section{Introduction}

Shales, despite displaying low porosity and permeability, have characteristic pore-size distributions (PSD) which tend to be complex in organic-rich shales because of the substantial contribution from porosity within the kerogen. The PSD complexity tends to increase in shales with high total organic carbon (TOC) and high levels of thermal maturity, as they have a wider range of pore sizes and greater pore volumes (Wood and Hazra, 2017). Pore-surface roughness of a shale's PSD, characterized by its fractal dimension, determines how much gas they can adsorb (Pfeifer et al., 1989). Fractal dimension is also strongly correlated with the ability of petroleum fluids to flow through the pore space (effective permeability) and, therefore, how much gas and/or oil could ultimately be produced and recovered through wellbores drilled into a shale formation (Alturki et al., 2014). Following the development of fractal theory, pioneered in the 1970s (Mandelbrot, 1975), the recognition that fractal dimension provided a valuable insight to the roughness of surfaces within porous media followed in the 1980s (Pfeifer, 1983, 1984). This characteristic was linked to the distinct molecular structure of the pore surfaces (Avnir et al., 1984). The fractal dimension scale was shown to vary between 2 (completely smooth) and 3 (extremely rough) (Avnir et al., 1992).

Traditionally, fractal dimension has been considered to be independent of the magnitude of the pores and believed to respond only to the roughness of the pore surfaces (Mahamud and Novo, 2008; Sakhee-Pour and Li, 2016). However, correlations have been observed between fractal dimension and key physical properties of certain organic-rich shales (e.g., TOC, Tmax, micro-pore surface area and volume) (Hazra et al., 2018; Liu et al., 2021). These findings suggest that the

\section{Yandy
Scientific}

Press
${ }^{*}$ Corresponding author.

E-mail address: dw@dwasolutions.com (D. A. Wood). 
nature of the PSD probably does impact fractal dimension but in relatively complex ways that require more detailed study. It is now generally accepted that fractal dimension values substantially influence fluid flow, storage and recovery in all types of low-permeability petroleum reservoirs (Cai et al., 2020). There is, therefore, a pressing need to better understand these relationships, and to establish whether these influences introduce uncertainties into the fractal dimension calculation methods applied to all types of tight reservoirs.

The interpretation of gas-adsorption isotherms provides a means to estimate fractal dimension values (Sahouli et al., 1996). The techniques are now widely applied to petroleumrich shales (Clarkson et al., 2013; Yang et al., 2014; Wood and Hazra, 2017; Hazra et al., 2018; Liu et al., 2021; Tian et al., 2021), and the influence of a shale's thermal maturity on fractal dimension recognised (Hazra et al., 2018; Gao et al., 2020). Shale fractal dimension values published have been derived almost exclusively based on the interpretation of low-pressure gas adsorption isotherms exploiting the FrenkelHalsey-Hill (FHH) model (Halsey, 1948; Hill, 1952; Greggs and Sing, 1982). Most of the isotherms evaluated to derive fractal dimension values are established from low-pressure nitrogen adsorption experiments (Li et al., 2016; Yang et al., 2016). Alternative fractal dimension calculation techniques to FHH have been proposed, and theoretically evaluated and tested via simulations. Two such techniques are those proposed by Neimark (1992) (NM method) and Wang and Li (1997) (WL method). These two alternative techniques have been evaluated in conjunction with the FHH technique for multiple samples of the Bakken Shale collected from across the Williston Basin (U.S.A.) and displaying a range of PSD (Liu et al., 2021). The results presented in that study highlighted differences between the fractal dimension values calculated by the NM, WL and FHH techniques, and that those differences are of greater magnitude in the shales with larger micro-pore areas and volumes.

This study applies the FHH, NM and WL fractal dimension calculation techniques to two generic shale types: 1) lowmicro-pore volume/low maturity shale (sample E); and 2) high-micro-pore volume/high maturity shale (sample F). It considers, the steps in their calculation and interpretation and highlights discrepancies and uncertainties associated with the fractal dimension values estimated by each method. In particular, the curve and line fitting procedures used for the methods are evaluated and ways to optimize and standardize their interpretation considered.

\section{Method}

\subsection{FHH fractal calculation technique}

The theory underpinning the FHH technique involves a proportionality between the adsorbate film that coats pore surfaces in the porous media sampled with the fractal dimension value of those surfaces. Assuming a capillary condensation adsorption regime, the FHH technique relates fractal dimension to the adsorbate volume measured at different relative pressures as part of the isotherm. This is usefully expressed in $\log -\log$ terms as the relationship between its two components then approximates a straight line (Pfeifer and Cole, 1990)

$$
\ln N=a-(3-D) \ln \mu
$$

where $N$ is the volume of adsorbate adsorbed by the sample, $a$ is constant, $D$ is fractal dimension, and $\mu$ is adsorption potential

$$
\mu=R T \ln \frac{P}{P_{0}}
$$

where $R$ is universal gas constant, $T$ is absolute temperature, $P$ is equilibrium pressure of the porous sample for each pressure increment measured as part of the isotherm, and $P_{0}$ is saturation pressure of nitrogen at $77 \mathrm{~K}$.

The ratio $P / P_{0}$ determines a dimensionless relative pressure, which is referred to here as $X$. By combining Eq. (1) and Eq. (2), the log-log relationship between $N$ and $X$ becomes

$$
\operatorname{Ln} N=a+(D-3) \ln (-\ln X)
$$

The FHH technique involves a cross-plot of $\ln N$ versus $\ln (-\ln X)$ with a slope fitted to the theoretically expected linear trend that is equal to $D$ minus 3 . Deriving fractal dimension from the FHH method is therefore straightforward, as long as the $\ln N$ versus $\ln (-\ln X)$ trend is linear. Unfortunately, for many shales this trend is found to be non-linear to varying degrees, especially in those shales with complex PSD (Hazra et al., 2018). Consequently deriving best-fit lines to non-linear trends leads to uncertainty in the FHH $D$ values derived for such shales.

\subsection{NM fractal calculation technique}

The NM technique (Neimark, 1992) considers the thermodynamics of the $N$ versus $X$ relationship to derive a value for $D$. This relates the surface area $(S)$ to pore radius $(r)$ and $D$

$$
\ln S=k-(D-2) \ln r
$$

where $k$ is constant, and $S$ is calculated with the Kiselev relationship (Kiselev and Pavlova, 1965)

$$
S(X)=\frac{R T}{\sigma} \int_{N(X)}^{N_{\max }} \ln X d N(X)
$$

where $N_{\max }$ is quantity of adsorbate adsorbed as $X$ approaches 1 , and $\sigma$ is adsorbate's surface tension.

A cross-plot of $N$ versus $\ln X$ defines a curve for each isotherm. Theoretically, that curved trend should approximate a power curve. The accuracy with which that trend can be fitted with a power curve, by establishing precise values for its slope and exponent, strongly influence the integral values of $\ln X$ determined.

The pore radius $r$ can be determined using the Kelvin relationship (Powles, 1985)

$$
r=\frac{-2 \sigma V_{L}}{R T \ln X}
$$

where $V_{L}$ is molar volume of liquid adsorbate. Eq. (6) essentially expresses the variations in vapor pressure at the curved liquid-vapor interface in the pore spaces. 
The $N$ versus $\ln X$ power curve relationship is expressed as Eq. (7), in which $N$ is measured in $\mathrm{m}^{3} / \mathrm{kg}$. $R, T$ and $\sigma$ are constants, and $\sigma$ for nitrogen in a liquid state near to its boiling point is $0.04624 \mathrm{~N} / \mathrm{m}$. The molar volume of liquid nitrogen $\left(V_{L N}\right)$ is derived by dividing its molar mass $(0.028$ $\mathrm{kg})$ by its liquid density $\left(808 \mathrm{~kg} / \mathrm{m}^{3}\right)$

$$
-\ln X=m N^{b}
$$

where $m$ is slope, $b$ is exponent.

Taking the $m$ and $b$ values from the "best" fit to the curve, this can be entered into an integral solution to Eq. (5), as expressed by Eq. (8) to determine $S\left(X_{N}\right)$ for each $N$ value from the isotherm.

$$
S\left(X_{N}\right)=\frac{m R T}{\sigma(b+1)}\left(N_{\max }^{b+1}-N^{b+1}\right)
$$

Entered into Eq. (6) as constant values, $\sigma, V_{L}, R$ and $T$ contribute to the $r$ versus $\ln X$ trend defined. However, the values of those terms do not affect the slopes of the "bestfit" curve to the adsorption isotherms; they only influence its intercept on a $\ln S$ versus $\ln X$ cross-plot.

Simulated comparisons of fractal dimension values calculated for a range of porous media using the FHH and NM techniques (Jaroniec, 1995; Wang and Li, 1997) concluded that they could be reconciled theoretically. The simulations revealed, however, that the fractal dimension values derived from the NM technique were only realistic (i. e., yielded a stable value between 2 and 3 ) for a limited portion of the simulated isotherms considered.

\subsection{WL fractal calculation technique}

The WL technique (Wang and $\mathrm{Li}, 1997$ ) was designed to provide a more robust thermodynamically derived value of fractal dimension than the NM technique. It also assumes that the fractal surface described can be considered as an inscribed equicurved surface with a variable mean curvature radius. The surface area can therefore be expressed in terms of $D$ and $r$

$$
S(r)=k_{0}^{D} r^{2-D} V^{\frac{D}{3}}
$$

where $k_{0}$ is a constant and $V$ is volume.

Assuming that the absorbate adsorbed by the sample is not compressed, $V$ becomes a simple function of $N(X)$

$$
V(X)=\left[N_{\max }-N(X)\right] V_{L}
$$

Entering $S(X)$ and $V(X)$ values into Eq. (9) results in

$$
\frac{-\int_{N(X)}^{N_{\max }} \ln X d N(X)}{r^{2}(X)}=\frac{k_{0}^{D} V_{L}^{\frac{D}{3}} \sigma}{R T}\left\{\frac{\left[N_{\max }-N(X)\right]^{\frac{1}{3}}}{r(X)}\right\}^{D}
$$

Wang and $\mathrm{Li}$ (1997) expressed the left-side of Eq. (11) as $A(X)$ and the right-side of Eq. (11) as $B(X)$

$$
A(X)=\frac{-\int_{N(X)}^{N_{\max }} \ln X d N(X)}{r^{2}(X)}
$$

$$
B(X)=\frac{\left[N_{\max }-N(X)\right]^{\frac{1}{3}}}{r(X)}
$$

By applying natural logarithms to $A(X)$ and $B(X)$, Eq. (11) can be simplified to

$$
\ln A(X)=l+D \ln B(X)
$$

where $l$ is a constant and $D$ is the slope of a linear equation.

Expressed as Eq. (14), and making the assumption of capillary condensation conditions (as is the case with the FHH technique), the WL technique establishes a series of $A(X)$ and $B(X)$ values to estimate $D$. As is the case with the NM technique, $r(X)$ is calculated with Eq. (6) and to generate a suite of $\ln X$ values related to specific $N$ values the power curve best-fit to the $N$ versus $\ln X$ trend is needed. The same best-fit solution with Eq. (7) used by the NM technique is used for the WL technique for this purpose. Eq. (15) expresses the integral solution to Eq. (12) for specific $N$ values

$$
A\left(X_{N}\right)=\frac{m\left(N_{\max }^{b+1}-N^{b+1}\right)}{(b+1) r^{2}\left(X_{N}\right)}
$$

A cross-plot of $\ln A$ and $\ln B$ closely approximates a straight line whatever power curve solution is applied. If the best-fit power curve of the $N$ versus $\ln X$ trend is good (i. e., very low residuals) then the slope of the $\ln A$ versus $\ln B$ trend should unequivocally provide an accurate value of $D$. The simulations presented by Wang and $\mathrm{Li}$ (1997) for a range of porous media, resulted in credible results, i. e., $2<D<3$, across a scale of 1 to $250 \mathrm{~nm}$. This suggests that the WL technique should be more robust than the NM technique when applied to organicrich shales. However, as will be shown in this study, the quality of the derived fractal dimension value is heavily influenced by the quality of the power curve fit to the $N$ versus $\ln X$ trend emanating directly from the recorded adsorption isotherm data points.

\subsection{Implementing three fractal dimension calculation techniques}

The calculations presented in this study relate to the evaluations of Eq. (3) for the FHH technique, Eqs. (6)-(8) for the NM technique, and Eqs. (6), (7), (13) and (15) for the WL technique.

FHH technique: Eq. (3) provides data points for the entire relative pressure range recoded as part of an adsorption isotherm. By cross-plotting $\ln N$ and $\ln (-\ln X)$ a trend is established that, theoretically, should be approximately linear with a negative slope. A "best-fit" line, based on linear regression, incorporating all data points provides the slope of that trend from which FHH $D$ is calculated by adding 3 to the negative slope value.

NM technique: Eqs. (6)-(8) need to be calculated for all data points of the adsorption isotherm. The power curve "bestfit" to the $-\ln X$ versus $N$ trend is essential to provide values $m$ and $b$ with which Eq. (6) and Eq. (8) are evaluated. Crossplotting $\ln S$ versus $\ln r$ for all data points should, theoretically, yield a linear trend with a negative slope. However, for most 
shales the $\ln S$ versus $\ln r$ trend is almost always curved, particularly for the high relative pressure data points. NM $D$ is derived by subtracting the negative slope of the best linear fit for that line from 2. The curvature of that line often leads to erroneous NM $D$ values being calculated if all the isotherm data points are considered.

WL technique: Eqs. (6), (7), (13) and (15) need to be calculated for all data points of the adsorption isotherm. The same power curve fit procedure used for the NM technique is also required for the WL technique. Cross-plotting $\ln A$ versus $\ln B$ for all data points should, theoretically, yield a linear trend with a positive slope, and, in practice for most shales, does so for $b$ and $m$ values that define the fitted power curve. Establishing a definitive best-fit line (by linear regression) through the $\ln A$ versus $\ln B$ trend is typically, therefore, straightforward, and can be achieved with very small residuals. The WL $D$ is then the slope of the best-fit line to the $\ln A$ versus $\ln B$ trend. However, the accuracy of the WL technique is not best assessed in terms of the linearity of the $\ln A$ versus $\ln B$ trend, rather, as will be illustrated, it is the precision with which a power curve can be fitted to the $-\ln X$ versus $N$ trend.

In order to determine the slopes $(m)$ and exponents $(b)$ of possible power-curve fits to the $-\ln X$ versus $\mathrm{N}$ trend, it is helpful to use an optimizer configured in different ways to establish a feasible range of possible fits. Various configurations can involve different statistical measures of accuracy as objective functions, e. g., root mean squared error (RMSE) and mean absolute error (MAE), and they can configure the power curve in log-log terms while applying the optimizer. These alternative optimizer configurations applied are described in section 3.2. It is informative to compare NM $D$ and WL $D$ values derived from different power curve fits with $\mathrm{FHH} D$ values.

To facilitate detailed comparisons between FHH $D$, NM $D$ and WL $D$ derived from the same isotherm data, three graphical displays are found to be particularly informative: i) cross-plot of $\ln N$ versus $\ln (-\ln X)$ highlighting the $\mathrm{FHH}$ "best-fit" line together with a line with a slope that would be required to match the WL $D$ value; ii) cross-plot of $\ln S$ versus $\ln r$ displaying all isotherm data points and the NM "best-fit" line through those points, together with a line with a slope that would be required to match the WL $D$ value; and iii) cross-plot of $-\ln X$ versus $N$ displaying the curved trend involving all isotherm data points and the selected "best-fit" power curve, together the curve fit that would be needed to reproduce $\mathrm{FHH}$ $D$. Considered together, these three cross-plots can identify consistencies and/or the nature of discrepancies between the FHH $D$, NM $D$ and WL $D$ techniques.

\subsection{Generic organic-rich shale adsorption considered}

To illustrate the FHH, NM and WL techniques applied to different types of organic-rich shale, two generic shales with characteristic isotherm shapes, Shales E and F, are evaluated (Fig. 1 and Tables 1 and 2). Shale E is selected to represent a sample with low to moderate thermal maturity with a relatively low micro-pore component to its PSD. On the other hand, shale $\mathrm{F}$ represents a sample of high thermal maturity with micro-porosity representing a substantial portion of its PSD (average pore radius $<10 \mathrm{~nm}$ ).

Fig. 1 highlights the larger pore volume of shale $\mathrm{F}$ accessible to low-pressure gas adsorption compared to shale E. The gradients associated with the isotherm curve on the $N$ versus $X$ cross-plot are slightly steeper for shale $\mathrm{F}$ than for shale $\mathrm{E}$, across the full range of relative pressures assessed.

\section{Results}

\subsection{Fractal dimensions derived for shales $\mathbf{E}$ and $\boldsymbol{F}$ using the three techniques}

The raw isotherm data plotted in Fig. 1 is listed in the first two columns of Tables 1 and 2. The results of Eq. (3) analysis of the isotherm data for cross-plotting $\ln N$ and $\ln (-\ln X)$ trend from which the FHH $D$ values are calculated are listed in columns 3 and 4 of Tables 1 and 2. The results Eqs. (6)-(8) analysis of the isotherm data for cross-plotting the $\ln S$ versus $\ln r$ trend, from which the NM $D$ values are calculated, are listed in columns 5 and 6 of Tables 1 and 2 . The results of Eqs. (6), (7), (3) and (15) analysis of that isotherm data for cross-plotting the $\ln A$ versus $\ln B$ trend, from which the WL $D$ values are calculated, are listed in columns 7 and 8 of Tables 1 and 2.

The $D$ values derived from the values presented in Tables 1 and 2, using all data points available across the full relative pressure range are listed in Table 3. It is apparent that from Tables 1 and 2. Table 3 that for shale $\mathrm{E}$ the FHH and WL calculated fractal dimension values are almost identical $(\sim 2.5)$, but the NM $D$ value is higher $(\sim 2.8)$. On the other hand, for shale $\mathrm{F}$, the FHH $D$ values $(\sim 2.8)$ is slightly higher than the WL $D$ value $(\sim 2.7)$ but the NM $D$ value is again higher $(>2.9)$.

Figs. 2(a)-2(d) display the key isotherm interpretation plots for shale E and Figs. 3(a)-3(d) display the key isotherm interpretation plots for shale $\mathrm{F}$. These plots help to explain the fractal dimension values derived by the different techniques (Table 3). The standard deviations of the three fractal dimension values derived is high for both shales in the context of the feasible fractal dimension scale range of 2 to 3 (i. e., $\sim 19 \%$

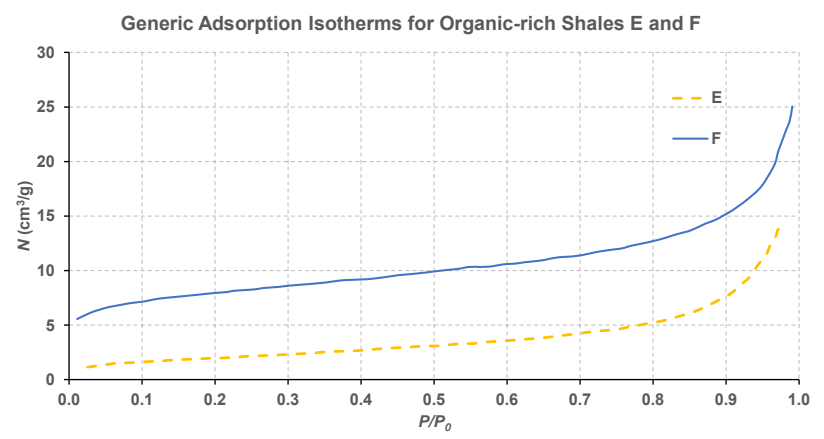

Fig. 1. Adsorption isotherms displayed for generic organic-rich samples E (low to moderate thermal maturity/low micro-pore porosity component) and $\mathrm{F}$ (high thermal maturity/high micro-pore volume) from data provided in Tables 1 and 2 . 
Table 1. Adsorption isotherm data and key calculated variables for generic organic-rich shale sample E (low to moderate thermal maturity/low micro-pore porosity component). These data are used to determine fractal dimension $(D)$. Note that $P / P_{0}$ is referred to as $X$ in the text and figures. N/A means "not applicable".

\begin{tabular}{|c|c|c|c|c|c|c|c|}
\hline$P / P_{0}$ & $N\left(\mathrm{~cm}^{3} / \mathrm{g}\right)$ & $\ln N$ & $\ln (-\ln X)$ & $\ln S$ & $\ln r$ & $\ln A$ & $\ln B$ \\
\hline 0.0252 & 1.1545 & -6.7641 & 1.3031 & 4.2918 & -20.4157 & 35.5875 & 18.9689 \\
\hline 0.0504 & 1.3665 & -6.5955 & 1.0944 & 4.1438 & -20.2071 & 35.0223 & 18.7548 \\
\hline 0.0756 & 1.5262 & -6.4849 & 0.9485 & 4.0447 & -20.0612 & 34.6314 & 18.6048 \\
\hline 0.1176 & 1.6877 & -6.3844 & 0.7608 & 3.9527 & -19.8735 & 34.1640 & 18.4128 \\
\hline 0.1429 & 1.7951 & -6.3227 & 0.6657 & 3.8954 & -19.7784 & 33.9165 & 18.3148 \\
\hline 0.1933 & 1.9574 & -6.2361 & 0.4969 & 3.8137 & -19.6096 & 33.4972 & 18.1416 \\
\hline 0.2395 & 2.1193 & -6.1567 & 0.3571 & 3.7374 & -19.4698 & 33.1413 & 17.9974 \\
\hline 0.2773 & 2.2280 & -6.1067 & 0.2489 & 3.6885 & -19.3616 & 32.8760 & 17.8861 \\
\hline 0.3214 & 2.3897 & -6.0366 & 0.1266 & 3.6191 & -19.2393 & 32.5619 & 17.7593 \\
\hline 0.3529 & 2.5500 & -5.9716 & 0.0406 & 3.5535 & -19.1533 & 32.3243 & 17.6687 \\
\hline 0.3950 & 2.6592 & -5.9297 & -0.0737 & 3.5105 & -19.0390 & 32.0528 & 17.5513 \\
\hline 0.4370 & 2.8730 & -5.8524 & -0.1889 & 3.4296 & -18.9238 & 31.7415 & 17.4299 \\
\hline 0.4874 & 3.0877 & -5.7803 & -0.3303 & 3.3524 & -18.7823 & 31.3814 & 17.2820 \\
\hline 0.5210 & 3.1959 & -5.7459 & -0.4277 & 3.3148 & -18.6850 & 31.1490 & 17.1814 \\
\hline 0.5546 & 3.3041 & -5.7126 & -0.5285 & 3.2780 & -18.5841 & 30.9106 & 17.0773 \\
\hline 0.5882 & 3.5171 & -5.6501 & -0.6337 & 3.2077 & -18.4790 & 30.6299 & 16.9655 \\
\hline 0.6492 & 3.8376 & -5.5629 & -0.8392 & 3.1063 & -18.2735 & 30.1176 & 16.7499 \\
\hline 0.6891 & 4.1559 & -5.4832 & -0.9878 & 3.0101 & -18.1249 & 29.7242 & 16.5909 \\
\hline 0.7269 & 4.4740 & -5.4095 & -1.1426 & 2.9175 & -17.9701 & 29.3219 & 16.4253 \\
\hline 0.7647 & 4.7921 & -5.3408 & -1.3158 & 2.8279 & -17.7969 & 28.8859 & 16.2410 \\
\hline 0.7983 & 5.2145 & -5.2563 & -1.4906 & 2.7123 & -17.6221 & 28.4208 & 16.0509 \\
\hline 0.8319 & 5.7416 & -5.1600 & -1.6928 & 2.5722 & -17.4199 & 27.8762 & 15.8285 \\
\hline 0.8655 & 6.4781 & -5.0393 & -1.9352 & 2.3807 & -17.1775 & 27.1999 & 15.5556 \\
\hline 0.8908 & 7.3184 & -4.9174 & -2.1569 & 2.1634 & -16.9558 & 26.5392 & 15.2955 \\
\hline 0.9181 & 8.5778 & -4.7586 & -2.4594 & 1.8273 & -16.6533 & 25.5981 & 14.9254 \\
\hline 0.9370 & 9.8363 & -4.6217 & -2.7318 & 1.4573 & -16.3808 & 24.6833 & 14.5683 \\
\hline 0.9454 & 10.6749 & -4.5399 & -2.8794 & 1.1736 & -16.2333 & 24.1045 & 14.3494 \\
\hline 0.9580 & 11.8804 & -4.4329 & -3.1483 & 0.6616 & -15.9644 & 23.0546 & 13.9403 \\
\hline 0.9685 & 13.1904 & -4.3283 & -3.4414 & -0.2679 & -15.6713 & 21.5389 & 13.3672 \\
\hline 0.9769 & 14.1861 & -4.2555 & -3.7559 & 1.0000 & -15.3568 & 1.0000 & N/A \\
\hline
\end{tabular}

Table 2. Summary of adsorption isotherm data and key calculated variables for generic organic-rich shale sample F. (high thermal maturity/high micro-pore volume). These data are used to determine fractal dimension $(D)$. Note that $P / P_{0}$ is referred to as $X$ in the text and figures. N/A means "not applicable".

\begin{tabular}{llllllll}
\hline$P / P_{0}$ & $N\left(\mathrm{~cm}^{3} / \mathrm{g}\right)$ & $\ln N$ & $\ln (-\ln X)$ & $\ln S$ & $\ln r$ & $\ln A$ & $\ln B$ \\
\hline 0.0110 & 5.5512 & -5.1937 & 1.5065 & 5.1368 & -20.6192 & 36.8394 & 19.3065 \\
0.0487 & 6.5551 & -5.0275 & 1.1062 & 4.6018 & -20.2189 & 35.5038 & 18.8886 \\
0.0801 & 6.9685 & -4.9664 & 0.9262 & 4.4040 & -20.0389 & 34.9461 & 18.7011 \\
0.1052 & 7.2047 & -4.9330 & 0.8119 & 4.2960 & -19.9245 & 34.6093 & 18.5823 \\
0.1429 & 7.5591 & -4.8850 & 0.6657 & 4.1400 & -19.7784 & 34.1611 & 18.4295 \\
0.1947 & 7.9134 & -4.8392 & 0.4926 & 3.9906 & -19.6052 & 33.6653 & 18.2495 \\
0.2276 & 8.1496 & -4.8098 & 0.3921 & 3.8944 & -19.5047 & 33.3681 & 18.1444 \\
0.2653 & 8.3858 & -4.7812 & 0.2828 & 3.8006 & -19.3955 & 33.0559 & 18.0305 \\
0.3014 & 8.6220 & -4.7534 & 0.1817 & 3.7093 & -19.2944 & 32.7624 & 17.9246 \\
0.3438 & 8.8583 & -4.7264 & 0.0655 & 3.6201 & -19.1782 & 32.4408 & 17.8035 \\
\hline
\end{tabular}


Table 2. Summary of adsorption isotherm data and key calculated variables for generic organic-rich shale sample F. (high thermal maturity/high micro-pore volume). These data are used to determine fractal dimension $(D)$. Note that $P / P_{0}$ is referred to as $X$ in the text and figures. N/A means "not applicable". (Continuation Table)

\begin{tabular}{llllllll}
\hline$P / P_{0}$ & $N\left(\mathrm{~cm}^{3} / \mathrm{g}\right)$ & $\ln N$ & $\ln (-\ln X)$ & $\ln S$ & $\ln r$ & $\ln A$ & $\ln B$ \\
\hline 0.3721 & 9.0945 & -4.7001 & -0.0114 & 3.5330 & -19.1013 & 32.1999 & 17.7218 \\
0.4082 & 9.2126 & -4.6872 & -0.1097 & 3.4902 & -19.0030 & 31.9604 & 17.6209 \\
0.4505 & 9.5669 & -4.6494 & -0.2265 & 3.3645 & -18.8861 & 31.6011 & 17.4966 \\
0.4945 & 9.8622 & -4.6190 & -0.3507 & 3.2628 & -18.7620 & 31.2510 & 17.3660 \\
0.5338 & 10.1575 & -4.5895 & -0.4655 & 3.1635 & -18.6472 & 30.9221 & 17.2447 \\
0.5636 & 10.3346 & -4.5723 & -0.5561 & 3.1050 & -18.5566 & 30.6825 & 17.1501 \\
0.5950 & 10.5709 & -4.5497 & -0.6554 & 3.0283 & -18.4573 & 30.4072 & 17.0454 \\
0.6232 & 10.7480 & -4.5330 & -0.7490 & 2.9717 & -18.3637 & 30.1633 & 16.9476 \\
0.6515 & 10.9843 & -4.5113 & -0.8475 & 2.8973 & -18.2652 & 29.8919 & 16.8436 \\
0.6954 & 11.3386 & -4.4795 & -1.0128 & 2.7878 & -18.0999 & 29.4519 & 16.6698 \\
0.7457 & 11.9291 & -4.4288 & -1.2260 & 2.6108 & -17.8867 & 28.8484 & 16.4419 \\
0.7708 & 12.2835 & -4.3995 & -1.3458 & 2.5074 & -17.7669 & 28.5054 & 16.3129 \\
0.8163 & 12.9921 & -4.3434 & -1.5948 & 2.3060 & -17.5178 & 27.8059 & 16.0449 \\
0.8399 & 13.4646 & -4.3077 & -1.7458 & 2.1751 & -17.3669 & 27.3731 & 15.8806 \\
0.8713 & 14.2913 & -4.2481 & -1.9819 & 1.9511 & -17.1307 & 26.6769 & 15.6197 \\
0.8964 & 15.0591 & -4.1958 & -2.2129 & 1.7474 & -16.8998 & 26.0112 & 15.3640 \\
0.9294 & 16.5354 & -4.1022 & -2.6137 & 1.3600 & -16.4990 & 24.8223 & 14.9099 \\
0.9655 & 19.6063 & -3.9319 & -3.3482 & 0.5138 & -15.7645 & 22.5071 & 14.0261 \\
0.9812 & 22.6772 & -3.7864 & -3.9624 & -0.6424 & -15.1503 & 20.1226 & 13.1343 \\
0.9906 & 25.0394 & -3.6873 & -4.6603 & 1.0000 & -14.4524 & 1.0000 & N/A \\
\hline
\end{tabular}

Table 3. Fractal dimensions calculated for the entire adsorption isotherm of two generic shales by the three methods using all data points presented in Tables 1 and 2 .

\begin{tabular}{lll}
\hline Technique & $D$ Shale E & $D$ Shale F \\
\hline FHH & 2.505 & 2.762 \\
NM & 2.813 & 2.944 \\
WL & 2.482 & 2.660 \\
Standard deviation & 0.185 & 0.144 \\
FHH-WL & 0.023 & 0.102 \\
\hline
\end{tabular}

of that range for shale $\mathrm{E}$ and $\sim 14 \%$ of that range for shale F). Note, however, that the difference between the FHH $D$ and WL $D$ value is quite small for shale $\mathrm{E}$ but substantial for shale F (Table 3).

In theory, applied to the isotherm data for a porous sample, these techniques should derive similar fractal dimension values. For shale $\mathrm{E}$ this is the case for the FHH and WL techniques. The $\ln N$ versus $\ln (-\ln X)$ cross-plot (Fig. 2(a)) establishes a clear linear trend yielding a slope from which the FHH $D$ value can be determined with little margin for error. The WL $D$ slope overlays that linear trend, as it should as WL $D \approx$ FHH $D$. The WL $D$ slope displayed in Fig. 2(a) is the slope that would be required on that plot to match the fractal dimension value calculated by the WL method.

Note that the low $P / P_{0}$ values ( $D 1$ fractal component portion of the isotherm $\left.0<P / P_{0}<0.5\right)$ are towards the right on the $\ln N$ versus $\ln (-\ln X)$ trend (Fig. 2(a)) and that is also the case for the $\ln A$ versus $\ln B$ trend (Fig. 2(d)). However, for Figs. 2(b) and 2(c), it is the high $P / P_{0}$ values (D2 fractal component portion of the isotherm $0.5<P / P_{0}<1$ ) that are positioned towards the right.

The $\ln S$ versus $\ln r$ trend is highly non-linear (Fig. 2(b)), as it is for many shales, making the best fit linear trend through it highly dependent on a few data points at the high relative pressure end of the trend. The WL slope is consistent with all but the ten or so data points representing the highest relative pressures. The WL $D$ slope displayed in Fig. 2(b) is the slope that would be required on that plot to match the fractal dimension value calculated by the WL method.

This suggests that by disregarding those data points an adjusted NM $D$ close to the WL $D$ value could be derived sts that by disregarding those data points an adjusted NM $D$ close to the WL $D$ value could be derived.

For Shale E, the power curve fits to the $-\ln X$ versus $N$ curved trend are very good and almost overlay the data points (Fig. 2(c)). Significantly, the power fit needed to generate the FHH $D$ value also overlays the data trend. Excel's "autofit" power curve does not fit the data quite as well as the optimum fit at the low pressure end of the trend. The linear fit of the $\ln A$ versus $\ln B$ trend (Fig. 2(d)) is based on a very high coefficient of determination $\left(\mathrm{R}^{2}=0.9997\right)$ generating confidence in the WL slope derived from it. 


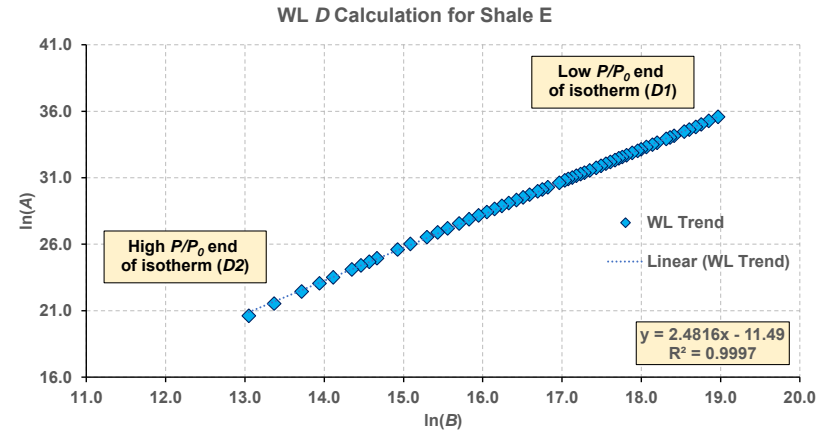

(a)

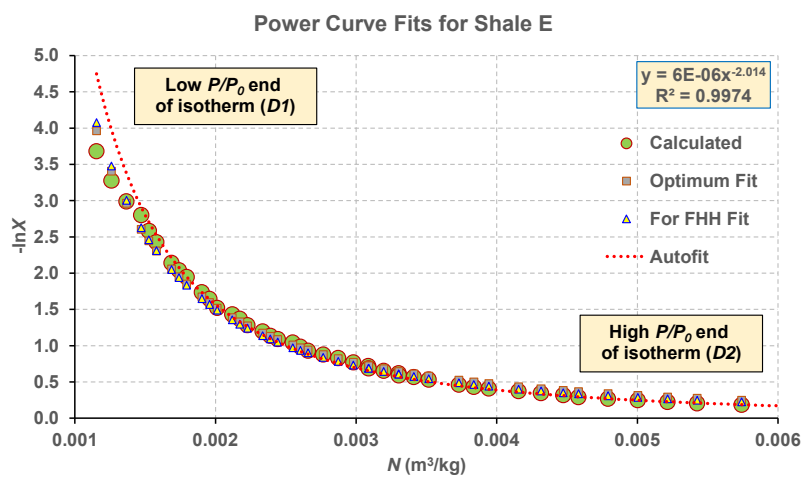

(c)

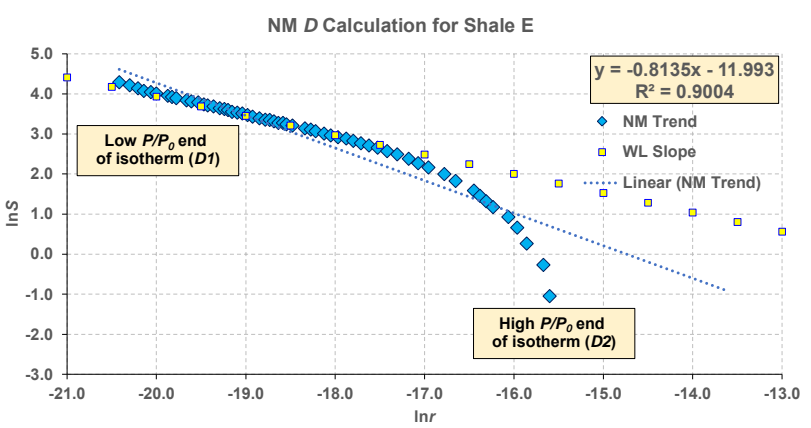

(b)

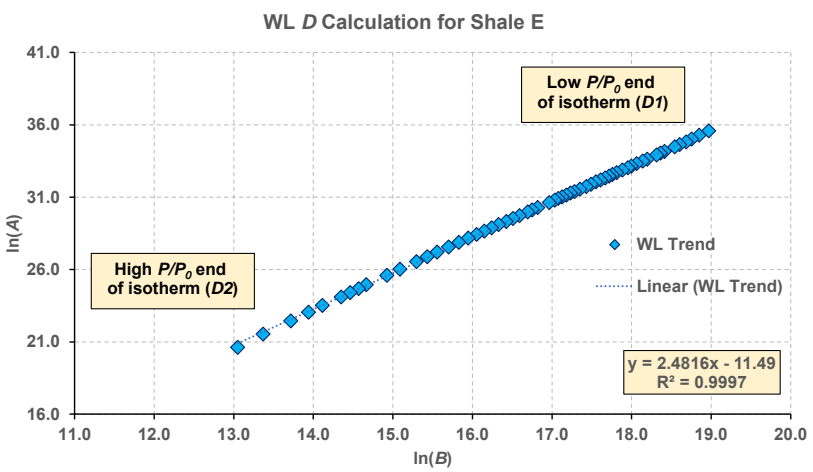

(d)

Fig. 2. Isotherm interpretation plots for shale E: (a) for FHH $D$ calculation with WL slope superimposed; (b) for NM $D$ calculation with WL slope superimposed; (c) power curve "best" fits for NM $D$ and WL $D$ calculations; and (d) WL $D$ slope calculation.

As the $D$ value differences suggest for the three techniques (Table 3), the trends defined by the shale $\mathrm{F}$ analysis are not so clear cut (Fig. 3). The data point trend on the $\ln N$ versus $\ln (-\ln X)$ cross-plot is subtly curved (Fig. 3(a)) and the FHH linear trend and the WL slope do not coincide. The WL slope tends to follow the low-relative-pressure part $(D 1)$ of that trend, whereas the FHH best-fit line is substantially influenced by the middle- and high-relative-pressure parts of the trend. The $\ln S$ versus $\ln r$ trend is curved upwards at the lowrelative pressure end $(D 1)$ of the trend and downwards at the high-relative-pressure (D2) end of the trend (Fig. 3(b)). This introduces uncertainties to the linear fit established through that line generating the NM $D$ value (2.944; Table 3). The WL slope follows the middle part of that trend, suggesting that data points at both the $D 1$ and $D 2$ ends of the Fig. $3 \mathrm{~b}$ trend would need to be disregarded to generate an adjusted NM $D$ value close to the WL $D$ value for Shale F. However, once data points are disregarded for the purpose of the NM $D$ calculation, particularly from both ends of the isotherm, it makes the NM $D$ calculation subjective and easy to manipulate to derive a desired fractal dimension value.

The various power-curve "best-fit" alternatives displayed in Fig. 3(c) do not precisely fit the $-\ln X$ versus $N$ curved trend for shale F. In particular, the power curve required for the WL $D$ to reproduce the FHH $D$ (triangles Fig. 3(c)) represents a relatively poor fit to that data trend. Several possible power curves can be used to approximate the $-\ln X$ versus $N$ curved trend, some better fitting the data at certain points along the trend. A different WL $D$ and NM $D$ value results for each of the slightly imperfect power curves fits, introducing substantial uncertainty into the WL $D$ and NM $D$ derivations. No matter what power curve fit is selected, the linear fit of the $\ln A$ versus $\ln B$ trend (Fig. 3(d)) generates a very high coefficient of determination $\left(\mathrm{R}^{2}>0.99\right)$ generating confidence. This highlights that it is the quality of the power curve fit used (Fig. 3(c)) rather than the $\mathrm{R}^{2}$ value in Fig. 3(d) that should determine the confidence placed in the WL $D$ value derived. For shales such as shale $\mathrm{F}$, it is not unusual for the calculations (based on all isotherm data points) to generate NM $D \gg \mathrm{FHH}$ $D>$ WL $D$.

\subsection{Optimizing the power curve fit to the $-\ln X$ versus $N$ curved trend}

Based on the results for shale F (Fig. 3(c)), it is appropriate to consider how best to optimize the curve fits for shales where the $-\ln X$ versus $N$ trend does not conform exactly to a power curve. Justifying the best-fit curve selected helps to improve confidence in the WL $D$ value derived. The standard non-linear "Solver" optimizers (FrontLine Solvers, 2021) forming part of Microsoft Excel ${ }^{\mathrm{TM}}$ spreadsheets are convenient optimizers to use as the other calculations and graphics required for the FHH, NM and WL $D$ calculations are readily performed in that software. These solvers can be configured and driven by Visual Basic for Application (VBA) coded macros, available in Excel, and conveniently operated from buttons placed on the appropriate spreadsheets. The Solver optimizers include Generalized Reduced Gradient (GRG) and an evolutionary non- 


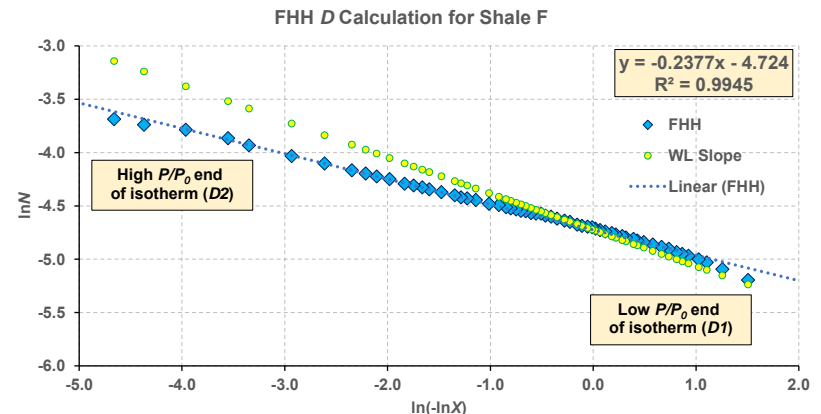

(a)

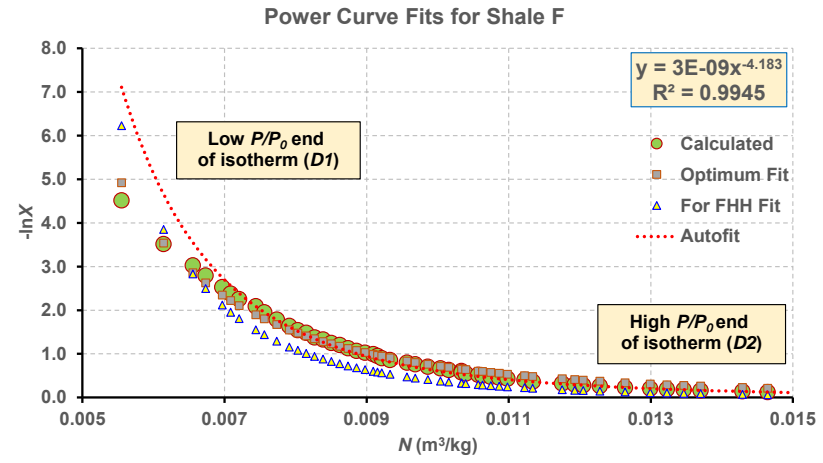

(c)

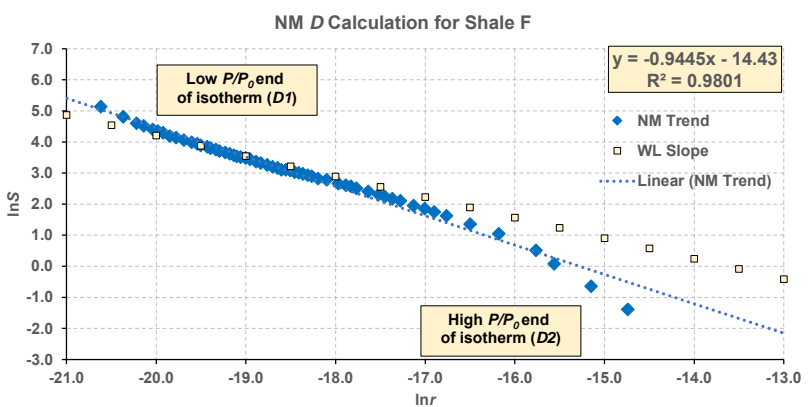

(b)

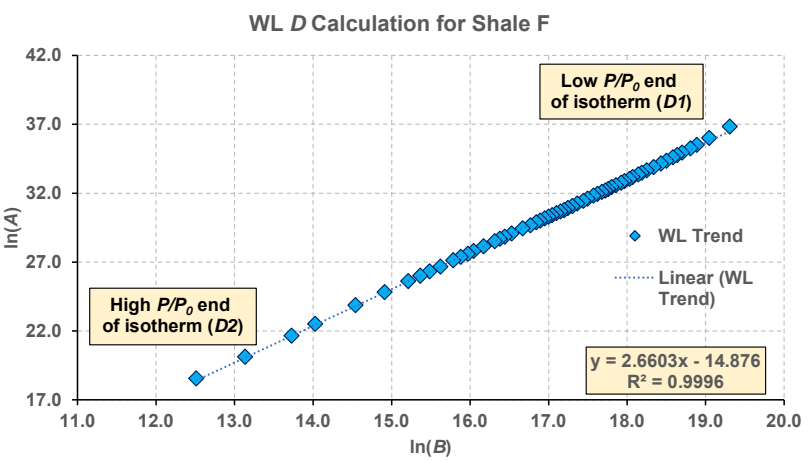

(d)

Fig. 3. Isotherm interpretation plots for shale F: (a) for FHH $D$ calculation with WL slope superimposed; (b) for NM $D$ calculation with WL slope superimposed; (c) power curve "best" fits for NM $D$ and WL $D$ calculations; and (d) WL $D$ slope calculation.

linear optimizer. Both of these optimizers operate sufficiently and rapidly for power-curve fitting with GRG working best in "multi-start" mode.

Six optimization options are configured to generate their own optimum best-fit power curves to the $-\ln X$ versus $N$ trend:

Opt 1. Find $b$ (exponent) and $m$ (slope) values (two unknowns in Eq. (7) by calculating $-\ln X$ values for all isotherm data point and minimizing the RMSE for the actual versus calculated $-\ln X$ values.

Opt 2. As for option 1 but disregarding a number of the lowest relative pressure data points. Here, the lowest four X values have been disregarded from the RMSE calculations.

Opt 3. Apply Excel's in-built power-fit trendline option to all isotherm data points forming the $-\ln X$ versus $N$ trend and taking the $b$ and $m$ values for that trendline. No RMSE values are revealed for the "autofit" curve provided by Excel.

Opt 4. As for Opt 1 but expressing Eq. (7) in a linear form by taking natural logarithms of both side as shown in Eq. (16), and using the logarithmic values for the RMSE objective function

$$
\ln (-\ln X)=\ln m+b \ln N
$$

Opt 5. As for Opt 4 but configuring the optimizer to optimize $\ln m$ rather than $m$.

Opt 6. As for Opt 5 but using MAE as the optimizer's objective function rather than RMSE.

All of the options, except Opt 2, use all the isotherm data records to derive their optimum power curve fits.
The fits for each of the optimization set-ups applied to shale F are illustrated in Fig. 4 for all isotherm data points, and the NM $D$ and WL $D$ values generated by them are listed in Table 4. The WL $D$ calculated values for these optimized fits range from 2.50 to 2.66 and the NM $D$ values range from 2.77 to 2.94. These ranges highlight the sensitivity of the calculations to the power curve fits. The details of each power-curve fit are better illustrated by displaying the $-\ln X$ versus $N$ trend on an expanded scale in two parts (Fig. 5). The Opt 1 fit is accurate for the low-relative-pressure end of the trend but fails to accurately fit the middle- and high-relative-pressure end of the trend. On the other hand, Opt 3 and Opt 5 fit the middleand high-relative-pressure end of the trend accurately but fail to fit the low-relative pressure end of the trend. The Opt 2, Opt 4 and Opt 6 fall between those extremes.

The configuration of Opt 1 means that its RMSE objective function gives more weight to the discrepancies at the high

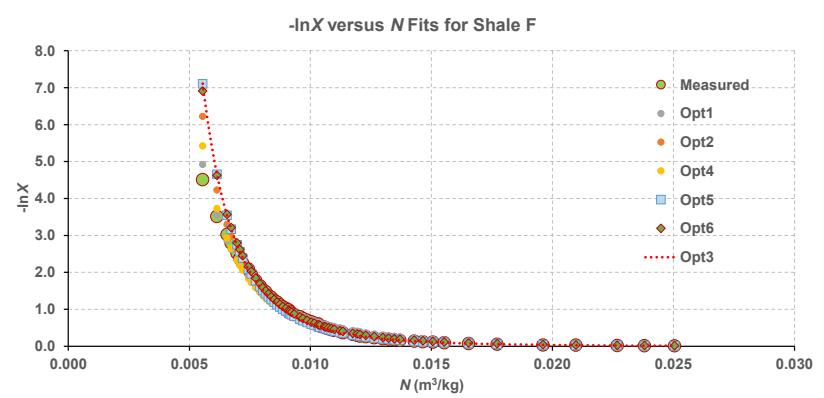

Fig. 4. Various imperfect power-curve fits to the $-\ln X$ versus $N$ trend for all data points of the Shale $\mathrm{F}$ isotherm. 
Table 4. NM $D$ and WL $D$ values calculated by applying various power curves fitted to the $-\ln X$ versus $N$ trend for the entire isotherm of shale $\mathrm{F}$ displayed in Fig. 4. The FHH $D$, for comparison, is calculated to be 2.762. N/D means not determined.

\begin{tabular}{lllllll}
\hline Technique & Opt 1 & Opt 2 & Opt 3 & Opt 4 & Opt 5 & Opt 6 \\
\hline NM & 2.769 & 2.874 & 2.944 & 2.852 & 2.944 & 2.904 \\
WL & 2.503 & 2.597 & 2.660 & 2.578 & 2.660 & 2.624 \\
\hline$b$ & -3.2588 & -3.8203 & -4.1834 & -3.7070 & -4.1834 & -3.9753 \\
$m$ & $2.19 \mathrm{E}-07$ & $1.50 \mathrm{E}-08$ & $2.60 \mathrm{E}-09$ & $2.36 \mathrm{E}-08$ & $2.60 \mathrm{E}-09$ & $7.46 \mathrm{E}-09$ \\
\hline RMSE & 0.1045 & 0.0326 & N/D & 0.1956 & 0.1049 & 0.1657 \\
\hline
\end{tabular}

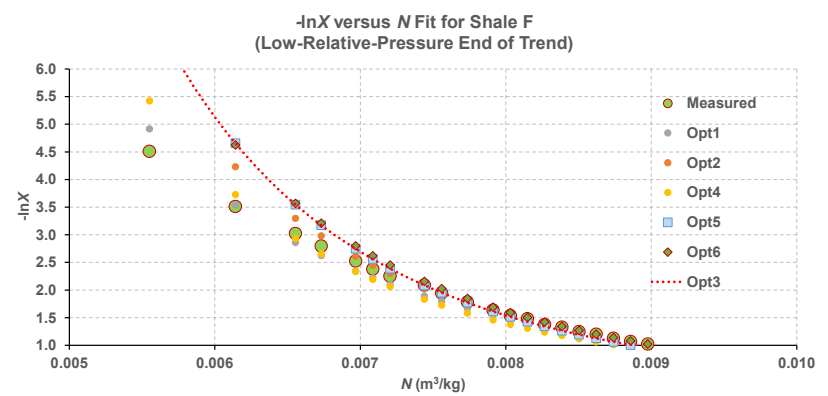

(a)

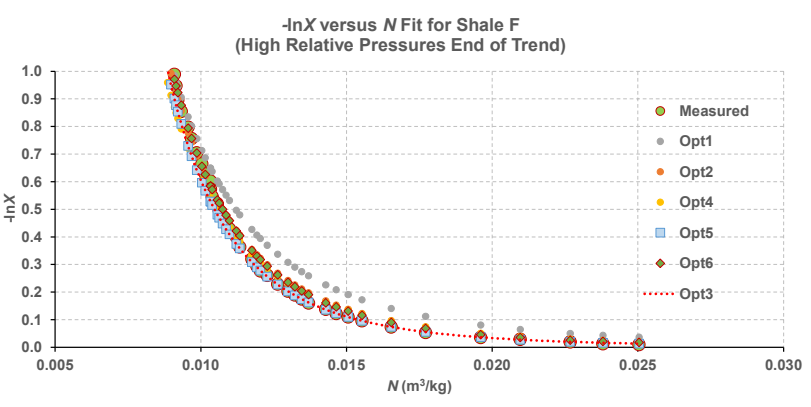

(b)

Fig. 5. Various imperfect power-curve fits to the $-\ln X$ versus $N$ trend for all data points of the Shale $\mathrm{F}$ isotherm.

$-\ln X$ valued points at the lower end of the relative pressure scale. Its "best" fits therefore tend to accentuate the importance of a few points in the $D 1$ portion of the isotherm, but do not accurately fit the $D 2$ portion of the isotherm. By ignoring a few points at the low-relative-pressure end of the trend Opt 2 in calculating RMSE Opt 2 provides a more balanced fit but involves subjectivity in deciding which points to include and ignore. By exploiting the logarithmic format of Eq. (7), Opt 4, Opt 5 and Opt 6 reduce the relative impact of the lowrelative-pressure end of the trend, because the range of the logarithmic values across the entire isotherm is smaller. As the $m$ values of the fits are very small (E-7 to E-9 for shale F) the optimizers are more effective by considering $\ln m$ rather than $m$. Consequently, Opt 5 and Opt 6 find their optimum values more easily than Opt 4.

The power curve fit established by Opt 5 is the same as that established by the Excel power-curve trendline (Opt 3). The Opt 6 fit is worth considering as it involves non-squared errors in the calculation of its MAD objective function. This can be useful in some cases where there a few very large values in the trend, as it reduces emphasis on those values, even in logarithmic form compared to the Opt 5 configuration. For shale F, it is the Opt 5 (and Opt 3) fit that is selected for the WL $D$ and NM $D$ values and the figures displayed in the four right-hand columns in Table 2 are calculated with that fit. None of these fitting options perfectly match the $-\ln X$ versus $N$ trend but Opt 5 takes better account of the $D 2$ portion of the isotherm than the other options for shale F. This may not be the case with other shales, making it appropriate to test several alternative fits before selecting the most reasonable.

There is still a difference between the Opt 5 WL $D$ value (2.66) and the FHH $D$ value (2.76) for shale F. Careful consideration of Fig. 3(a) helps to explain the reason for this difference. FHH $D$ derived from Fig. 3(d) as the slope of the best-fit line to that trend is clearly being strongly influenced by the high-relative-pressure component (left end) of that trend. On the other hand, the Opt $5 \mathrm{WL} D$ value, from its slope superimposed on Fig. 3(a) is mainly influenced by the lowrelative-pressure (right end) of that trend. As the trend of the actual data in Fig. 3(a) is curved (not linear) and the selected fit for Opt 5 WL (Fig. 4) is not a perfect fit, it is difficult to decide which of the two $D$ values is the most valid (FHH $D$ or WL $D$ ) for shale F. This comparison highlights the uncertainties involved in deriving fractal dimension values for shales such as shale $\mathrm{F}$. If there is no perfect power-curve fit to the $-\ln X$ versus $N$ trend, and the $\ln N$ versus $\ln (-\ln X)$ trend is not linear, as is the case for shale $F$, then it seems safer to quote a range for fractal dimension with the best-fit FHH $D$ and WL $D$ considered as likely limits of that range.

\section{Discussion}

It is also common practice to consider fits to two parts of an adsorption isotherm to obtain $D 1$ and $D 2$ fractal dimension estimates relating to the low- and high-relative-pressure portions of the isotherm respectively; where $D 1$ is calculated using data points conforming to $0<P / P_{0}<0.5$, and $\mathrm{D} 2$ is calculated using data points conforming to $0.5 \leqslant P / P_{0}<1.0$. This analysis has been conducted for shale $F$, and the power curve fitting option and their derived NM and WL fractal dimensions results are presented in Table 5 for $D 1$ and Table 6 for $D 2$.

For the $D 1$ portion of the Shale $\mathrm{F}$ isotherm, the $-\ln X$ versus $N$ trend cannot be fitted with a power curve precisely. On the other hand, for the $D 2$ portion of the isotherm, the 
Table 5. NM $D$ and WL $D$ values calculated by applying various power curves fitted to the low-relative-pressure portion of the $-\ln X$ versus $N$ trend $(D 1)$ for shale F. The FHH D1, for comparison, is calculated to be 2.708 . N/D means not determined.

\begin{tabular}{lllllll}
\hline Technique & Opt 1 & Opt 2 & Opt 3 & Opt 4 & Opt 5 & Opt 6 \\
\hline NM & 3.949 & 4.049 & 3.980 & 4.090 & 4.007 & 4.053 \\
WL & 2.649 & 2.714 & 2.669 & 2.740 & 2.687 & 2.716 \\
\hline$b$ & -3.0616 & -3.6316 & -3.2371 & -3.8565 & -3.3963 & -3.6507 \\
$m$ & $5.93 \mathrm{E}-07$ & $3.77 \mathrm{E}-08$ & $2.52 \mathrm{E}-07$ & $1.18 \mathrm{E}-08$ & $1.15 \mathrm{E}-07$ & $3.45 \mathrm{E}-08$ \\
\hline RMSE & 0.0700 & 0.0166 & N/D & 0.06679735 & 0.0317 & 0.0433 \\
\hline
\end{tabular}

Table 6. NM $D$ and WL $D$ values calculated by applying various power curves fitted to the high-relative-pressure portion of the $-\ln X$ versus $N$ trend $(D 2)$ for shale F. The FHH D2, for comparison, is calculated to be 2.775. N/D means not determined.

\begin{tabular}{lllllll}
\hline Technique & Opt 1 & Opt 2 & Opt 3 & Opt 4 & Opt 5 & Opt 6 \\
\hline NM & 3.086 & 3.088 & 3.088 & 3.086 & 3.088 & 3.096 \\
WL & 2.658 & 2.660 & 2.660 & 2.658 & 2.660 & 2.666 \\
\hline$b$ & -4.4304 & -4.4414 & -4.4424 & -4.4310 & -4.4424 & -4.4845 \\
$m$ & $9.10 \mathrm{E}-10$ & $8.66 \mathrm{E}-10$ & $8.60 \mathrm{E}-10$ & $9.43 \mathrm{E}-10$ & $8.60 \mathrm{E}-10$ & $7.14 \mathrm{E}-10$ \\
\hline RMSE & 0.0063 & 0.0060 & N/D & 0.0495 & 0.0385 & 0.0395 \\
\hline
\end{tabular}

$-\ln X$ versus $N$ trend can be fitted with a power curve precisely. This is apparent from the Tables 5 and 6 results. For $D 1$, none of the optimizers fits precisely overlay the $-\ln X$ versus $N$ trend, and the fits obtained suggest a substantial range of WL $D 1$ values (2.649 to 2.740). The Opt 2 and Opt 4 power-curve fits yield WL $D 1$ values that are closest to the FHH $D 1$ value and visually provide the most balanced fits to the data. For $D 2$, all of the optimizers fits overlay $-\ln X$ versus $N$ trend quite well, and, consequently, the fits suggest a relatively narrow range of WL $D 2$ values (2.658 to 2.666).

The agreement between the WL $D 1$ (2.72) and FHH D1 (2.71) is very good for Shale F. Despite the good power curve fit for the $D 2$ portion of the isotherm, there is, however, a clear discrepancy between the WL D2 (2.66) and FHH D2 (2.78) values derived for Shale F. These relationships are well illustrated in the $\ln N$ versus $\ln (-\ln X)$ cross-plots displayed in Fig. 6.

Whereas the WL slope overlays the FHH best-fit line for $D 1$ in Fig. 6(a), the WL D2 slope is clearly steeper than the FHH best-fit line for $D 2$ in Fig. 6(b). As for the full isotherm interpretation for shale $\mathrm{F}$, this $D 2$ discrepancy between the FHH and WL calculations seems to be best explained by FHH being more influenced by the higher-relative-pressure data points and WL being more influenced by the lowestrelative-pressure data points in the $D 2$ isotherm. Again this suggests, because of the slightly curved nature of the data points in Fig. 6(b) that the FHH D2 and WL D2 values are probably best considered as the limits of a range of uncertainty in the $D 2$ value for shale $\mathrm{F}$.

Fig. 7 displays on $\ln S$ versus $\ln r$ cross-plots for the relationship between the NM and WL slopes for the $D 1$ and $D 2$ isotherms of shale F. For the D1 isotherm (Fig. 7(a)), the WL slope aligns with those data points in the middle of the isotherm and is substantially lower than the slope of the best fit line through all data point used to derive NM $D 1$. For the $D 2$ isotherm (Fig. 7(b)), the WL slope aligns with those data points at the low-relative-pressure end of the isotherm and, also, is substantially lower than the slope of the best fit line through all data point used to derive NM D2. This provides further evidence that the WL $D 2$ value for shale $\mathrm{F}$ is more strongly influenced by the low-relative pressure end of the $D 2$ isotherm. It also explains why the WL $D 2$ value derived is lower than the FHH D2 value.

The analysis presented suggests that there is worthwhile information to be extracted by conducting fractal analysis from low-pressure gas adsorption isotherms using all three of the FHH, NM and WL techniques in an integrated analysis. By doing so it is possible to identify and justify the most credible specific $D, D 1$ and/or $D 2$ values or range of possible values based on the uncertainties exposed in the isotherm trends. Historically, researchers have relied almost exclusively on the FHH technique applying linear fits to $\ln N$ versus $\ln (-\ln X)$ plot trends, even if those trends are curved, to determine fractal dimension values. By carefully considering the trends defined by the $\ln N$ versus $\ln (-\ln X), \ln S$ versus $\ln r,-\ln X$ versus $N$ plots, and $\ln A$ versus $\ln B$ cross-plots and interrogating the linear and power curve fits to those trends a greater confidence in the fractal dimension values (or ranges of values) can be attained for a wide range of organic-rich shales. Although the NM $D$ values derived are considered less credible than the FHH and WL values, when all data points of the isotherm are considered, the $\ln S$ versus $\ln r$ trend used in the NM $D$ derivation is useful for providing insight to the portion of the isotherm best described by the WL $D$ value. Overlaying the WL slope on the $\ln S$ versus $\ln r$ trend and the $\ln N$ versus $\ln (-\ln X)$ leads to a clearer understanding of the interrelationships between the fractal dimension calculation techniques. As does a detailed analysis with optimizers of the 


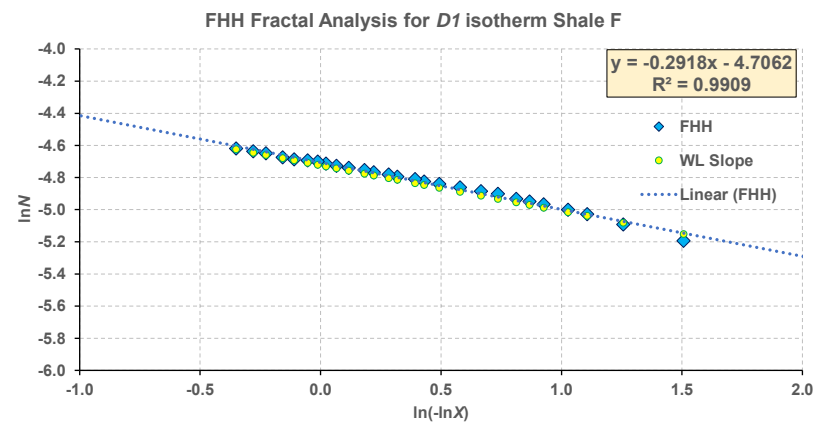

(a)

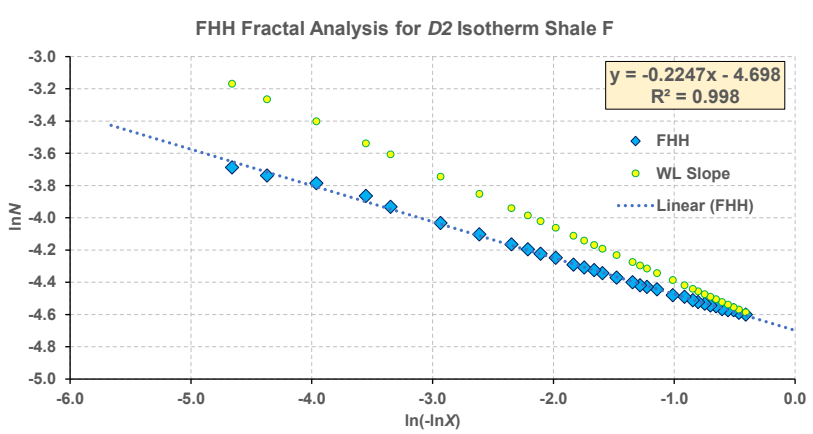

(b)

Fig. 6. $D 1$ and $D 2$ Isotherm interpretation plots for shale F showing FHH linear fits with the WL slope superimposed.

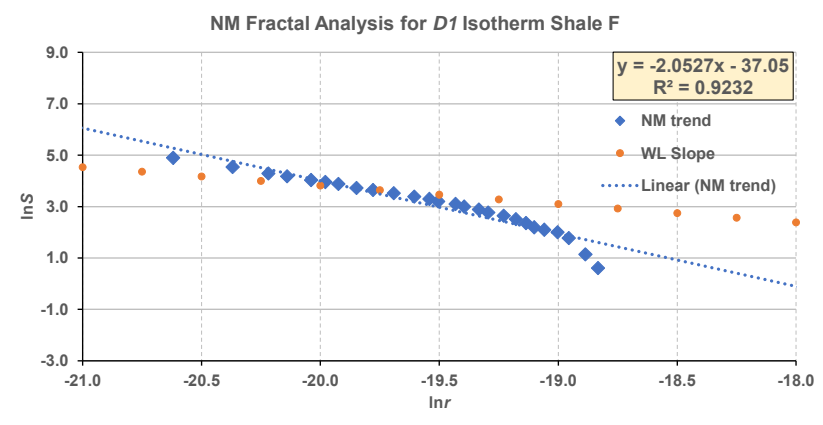

(a)

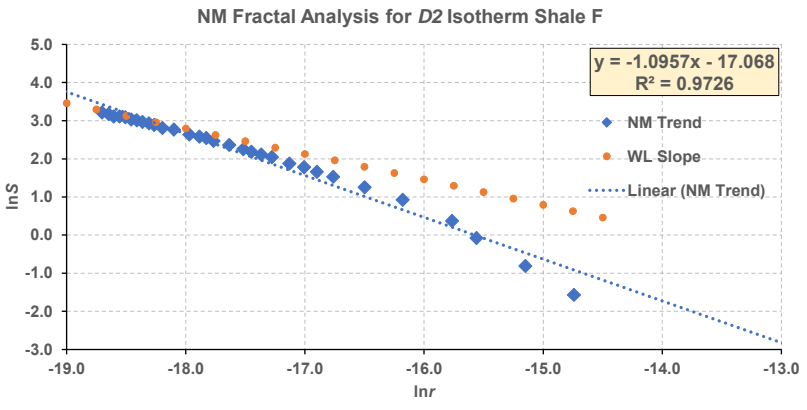

(b)

Fig. 7. $D 1$ and $D 2$ Isotherm interpretation plots for shale F showing NM linear fits with the WL slope superimposed.

quality of the power-curve fits to the $-\ln X$ versus $N$ curved trends.

Values of fractal dimension for organic-rich shales are important because they have a direct bearing on the ability of those formations to store and produce natural gas and gas liquids. There is a need to calculate these values as accurately as possible and to be able to justify with confidence the fractal dimension values, or value range, selected from the analysis. Using the FHH techniques in isolation is fraught with uncertainties. These uncertainties can be more clearly revealed by conducting more comprehensive analysis that integrates FHH, NM and WL techniques. As described, more reliable and consistent fractal dimension values (or ranges of values) can be derived from such an integrated analytical approach.

Irrespective of which type of shale is being evaluated, there is value in deriving and comparing both the FHH and WL $D$ values before selecting an appropriate fractal dimension value for the sample. The comparison is best performed using the $\ln N$ versus $\ln (-\ln X), \ln S$ versus $\ln r,-\ln X$ versus $N$ plots, and $\ln A$ versus $\ln B$ cross-plots. For shales generating a nearlinear trend involving all their isotherm data points on the $\ln N$ versus $\ln (-\ln X)$ cross-plot, the FHH $D$ value is likely to be realistic and agree reasonably closely with the WL $D$ value. On the other hand, for shales generating a non-linear trend involving all their isotherm data points on the $\ln N$ versus $\ln (-\ln X)$ cross-plot, the FHH $D$ value is less likely to be realistic. Shales with high thermal maturity and with microporosity representing a substantial portion of its PSD are more likely to generate the latter type of isotherm. For such shales, both FHH $D$ and WL $D$ should be considered. If it is not possible to justify one or other of those values, and there is a significant difference between their values, the fractal dimension should be quoted as a range between the FHH and WD values.

There is important ongoing research being conducted that is attempting to relate fractal dimension values of organic-rich shales to a range of their underlying rock properties (PSD, thermal maturity, organic content, organic mineralogy, etc.). To do this effectively accurate and credible fractal dimension values need to be derived.

\section{Conclusions}

Fractal dimensions of organic-rich shales are routinely calculated by applying the FHH technique to low-pressure gas adsorption isotherms. However, such shales tend to have complex and heterogeneous pore-size distributions with the micro-pore components varying substantially with their thermal maturity and organic content. Trends that should be linear for definitive FHH $D$ determination are frequently observed to be curved, leading to uncertainties in the slopes of the "best-fit" lines through the data from which the fractal dimension values are determined. Fractal dimension can also be determined from shale adsorption isotherm data using the techniques of NM and WL, but the fractal dimension values they determine are frequently different from the $\mathrm{FHH}$ $D$ values. Using generic adsorption isotherms from two shales, sample $\mathrm{E}$ of low thermal maturity and with a low micro-pore components to its PSD, and sample F of high thermal maturity 
and with a high micro-pore components to its PSD, the fractal dimension calculations of the FHH, NM and WL techniques can be usefully compared. The comparisons reveal and explain the differences in the fractal dimension values they generate, which are shown to be mainly due to line and curve fitting uncertainties, particularly for shales such as F.

The NM $D$ values using all data points from the isotherms are found to be systematically higher than the FHH $D$ and WL D. To derive comparable fractal dimension values by the NM technique typically up to fifteen of the data points at the highrelative-pressure end of the isotherms, and sometimes a few from the low-relative-pressure end, need to be disregarded for line fitting purposes. This makes the NM method somewhat subjective, less reliable and more focused on the low-relativepressure end of the isotherm.

A power curve fit is required for the NM and WL techniques. For shales such as $\mathrm{E}$ the isotherm data typically conform precisely to power curves, whereas for shales such as $F$ they often do not. Where there are uncertainties in the power-curve fit, applying optimizers configured with different objective functions helps to select more balanced approximate fits to the isotherm data. This can lead to closer agreement between the FHH $D$ and WL $D$ values. Where discrepancies exist, they are shown to be a consequence of the WL technique being more influenced by data points at the low-relativepressure end of the isotherm, in contrast to the FHH techniques which, in curved $\ln N$ versus $\ln (-\ln X)$ isotherm data trends is more influenced by the high-relative-pressure end of the isotherm.

A case is made for calculating fractal dimension with all three techniques in an integrated analysis and comparing the fractal dimension values derived. In particular, superimposing the slope equivalent to the WL $D$ value on the FHH $D$ and NM $D$ calculation cross-plots reveals, and helps to explain, relationships between the fractal dimension values suggested by each method. Taking such an approach enables the selection of a meaningful fractal dimension value to be made with more confidence. For some isotherms uncertainties exist regarding the fractal dimension value from which technique is the most representative to use for the sample. In such cases, it is better to quote the fractal dimension value as a possible range rather than as a single value. That range should typically incorporate both FHH $D$ and WL $D$ values.

\section{Acknowledgement}

Many thanks to Kouqi Liu for clarifying aspects of the calculation methods used in (Liu et al., 2021) and Jianchao Cai and his associates (Tian et al., 2021) for providing some useful additional insights with respects of fractal dimensions and their analysis.

\section{Note}

Complete versions of Tables 1 and 2 are available for downloaded in an Excel file [https://doi.org/10.46690/ager.2021.02.05].

\section{Conflict of interest}

The authors declare no competing interest.

Open Access This article is distributed under the terms and conditions of the Creative Commons Attribution (CC BY-NC-ND) license, which permits unrestricted use, distribution, and reproduction in any medium, provided the original work is properly cited.

\section{References}

Alturki, A. A., Maini, B. B., Gates, I. D. The effect of wall roughness on two-phase flow in a rough-walled Hele-Shaw cell. Journal of Petroleum Exploration and Production Technology, 2014, 4(4): 397-426.

Avnir, D., Farin, D., Pfeifer, P. Molecular fractal surfaces. Nature, 1984, 308: 261-263.

Avnir, D., Farin, D., Pfeifer, P. A discussion of some aspects of surface fractality and of its determination. New Journal of Chemistry, 1992, 16(4): 439-449.

Cai, J., Zhang, L., Wei, W. Modelling of Flow and Transport in Fractal Porous Media. Amsterdam, The Netherlands, Elsevier, 2020.

Frontline Solvers. Excel Solver-Optimization Methods, 2021.

Gao, Z., Fan, Y., Xuan, Q., et al. A review of shale pore structure evolution characteristics with increasing thermal maturities. Advances in Geo-Energy Research, 2020, 4(3): 247-259.

Gregg, S. J., Sing, K. S. W. Adsorption, Surface Area, and Porosity. 2nd ed. New York, USA, Academic Press, 1982.

Halsey, G. Physical adsorption on non-uniform surfaces. Journal Chemical Physics, 1948, 16(10): 931-937.

Hazra, B., Wood, D. A., Kumar, S., et al. Fractal disposition, porosity characterization and relationships to thermal maturity for the Lower Permian Raniganj basin shales, India. Journal of Natural Gas Science and Engineering, 2018, 59: 452-465.

Hill, T. L. Theory of physical adsorption. Advanced in Catalysis, 1952, 4: 211-258.

Jaroniec, M. Evaluation of the fractal dimension from a single adsorption isotherm. Langmuir, 1995, 11: 2316-2317.

Kiselev, A. V., Pavlova, L. F. Use of general equations of isotherms for the adsorption of benzene-n-hexane solutions on the adsorbents of various characters. Russian Chemical Bulletin, 1965, 14: 15-23.

Li, A., Ding, W., He, J., et al. Investigation of pore structure and fractal characteristics of organic-rich shale reservoir: A case study of Lower Cambrian Qiongzhusi formation in Malong block of eastern Yunnan Province, South China. Marine Petroleum Geology, 2016, 70: 46-57.

Liu, K., Ostadhassan, M., Jang, H. W., et al. Comparison of fractal dimensions from nitrogen adsorption data in shale via different models. Royal Society of Chemistry Advances, 2021, 11: 2298-2306.

Mahamud, M. M., Novo, M. F. The use of fractal analysis in the textural characterization of coals. Fuel, 2008, 87(2): 222-231.

Mandelbrot, B. B. Les objects fractals: Forme, hasard et dimension. Paris, France, Flammarion, 1975.

Neimark, A. New approach to the determination of the surface 
fractal dimension of porous solids. Physica A, 1992, 191(1-4): 258-262.

Pfeifer, P. Chemistry in noninteger dimensions between two and three. I. Fractal theory of heterogeneous surfaces. The Journal of Chemical Physics, 1983, 79: 3558.

Pfeifer, P. Fractal dimension as working tool for surface roughness problems. Applications of Surface Science, 1984, 18(1-2): 146-164.

Pfeifer, P., Cole, M. W. Fractals in surface science: Scattering and thermodynamics of adsorbed films II. New Journal of Chemistry, 1990, 14(3): 221-232.

Pfeifer, P., Wu, Y., Cole, M. W., et al. Multilayer adsorption on a fractally rough surface. Physical Review Letters, 1989, 62(17): 1997-2000.

Powles, J. G. On the validity of the Kelvin equation. Journal of Physics A: Mathematical and General, 1985, 18(9): 1551.

Sahouli, B., Blacher, S., Brouers, F. Fractal surface analysis by using nitrogen adsorption data: The case of the capillary condensation regime. Langmuir, 1996, 12(11): 28722874.

Sakhaee-Pour, A., Li, W. Fractal dimensions of shale. Journal of Natural Gas Science and Engineering, 2016, 30: 578582 .
Tian, Z., Wei, W., Zhou, S., et al. Experimental and fractal characterization of the microstructure of shales from Sichuan Basin, China. Energy \& Fuels, 2021, 35 (5): 3899-3914.

Wang, F., Li, S. Determination of the surface fractal dimension for porous media by capillary condensation. Industrial \& Engineering Chemistry Research, 1997, 36(5): 15981602.

Wood, D. A., Hazra, B. Characterization of organic-rich shales for petroleum exploration \& exploitation: A reviewPart 1: bulk properties, multi-scale geometry and gas adsorption. Journal of Earth Science, 2017, 28(5): 739757.

Yang, F., Ning, Z., Liu, H. Fractal characteristics of shales from a shale gas reservoir in the Sichuan Basin, China. Fuel, 2014, 115: 378-384.

Yang, R., He, S., Yi, J., et al. Nano-scale pore structure and fractal dimension of organic-rich Wufeng-Longmaxi shale from Jiaoshiba area, Sichuan Basin: Investigations using FE-SEM, gas adsorption and helium pycnometry. Marine and Petroleum Geology, 2016, 70: 27-45. 